\title{
Measurement of plasma current in Tokamaks using an optical fibre reflectometry technique
}

\author{
Marc Wuilpart, Willem Leysen, Andrei Gusarov, Philippe Moreau, and Patrice Mégret
}

\begin{abstract}
An optical time-domain reflectometer sensitive to the polarization of light is proposed for the measurement of plasma current in the Tore Supra fusion reactor. The measurement principle relies on the Faraday effect i.e. on the generation of a circular birefringence along an optical fiber subject to an axial magnetic field. The circular birefringence induces a polarization rotation that can be mapped along the fiber thanks to an opticaltime domain reflectometer followed by an linear polarizer. A proper fitting of the measurement trace then allows determining the applied plasma current. The sensor has been experimentally validated on the Tore Supra tokamak fusion reactor for a plasma current range going from 0.6 to 1.5 MA. A maximum error of $13.50 \%$ has been observed for the lowest current.
\end{abstract}

Index Terms-Optical time-domain reflectometry, Faraday effect, current sensing, polarimetry.

\section{INTRODUCTION}

I $\mathrm{N}$ tokamak fusion reactors, magnetic fields are used to squeeze the plasma in the shape of a torus [1]. In practice, it is fundamental to properly monitor the intensity of the plasma current in order to evaluate the plasma stability. Nowadays, the measurement of the plasma current is performed by means of various kinds of inductive coils (Rogowski, pick-up coils) [2]. Since these sensors require a temporal integration to calculate the value of the plasma current, they are prone to drift during long plasma discharges. Moreover the radiation load induces spurious electrical effects like radiationinduced conductivity, radiation-induced electrical degradation and radiation-induced electromotive force that perturb the proper operation of electrically-based current sensors [3].

FOCS (Fiber-optic Current Sensors) [4] constitute an alternative. They are based on the monitoring of the state of polarization (SOP) rotation undergone by a lightwave propagating in an optical fiber. In the presence of a current surrounded by the fiber, the magnetic field aligned with the fiber axis has for effect to rotate the light SOP via the so-called Faraday effect. From the rotation angle, it is possible to retrieve the surrounded current. This approach presents two drawbacks in the field of tokamak fusion reactor diagnosis. First, the FOCS only measures the SOP rotation within the range $0-2 \pi$. This is problematic for high plasma current measurement like in the future ITER (20 MA). Second, the presence of a lead fiber (fiber section situated between the interrogating device and the loop surrounding the vacuum vessel) deteriorates the

Marc Wuilpart and Patrice Mégret are with the Department of Electromagnetism and Telecommunications, University of Mons, Mons, Belgium e-mail: marc.wuilpart@umons.ac.be

Andrei Gusarov and Willem Leysen is with the Belgian Nuclear Research Center SCK-CEN, Mol, Belgium.

Philippe Moreau is with the CEA (IRFM), Saint-Paul-lez-Durance, France.
FOCS performance [6]. To eliminate these issues, a POTDR (Polarization Optical Domain Reflectometer) can be used since a POTDR trace contains information about the SOP spatial evolution along the fibre. Such an approach was proposed in [5] where currents up to $2.5 \mathrm{kA}$ were tested. The drawback of this technique is to require the full measurement of the backscattered SOP.

In this paper, we summarize the results obtained when using a simple approach (commercially available OTDR followed by a linear polarizer) for plasma current measurement. The sensor has been experimentally validated on the Tore Supra tokamak fusion reactor of the CEA (Cadarache, France) for a plasma current range going from 0.6 to $1.5 \mathrm{MA}$.

The next section of this paper described the principle of the POTDR approach. In Section III, the modeling of the sensor is described and the method to extract the plasma current is explained. Finally, section IV describes the experimental results obtained on the Tore Supra fusion reactor. Let us note that a theoretical background describing polarization effects in optical fiber and their modeling using the Jones formalism can be found in [11].

\section{MEASUREMENT PRINCIPLE AND SIGNAL ANALYSiS}

The measurement scheme is depicted in figure 1. A photoncounting optical time domain reflectometer launches optical pulses into the lead and sensing fibers (the sensing fiber is the fiber loop placed around the vacuum vessel) via a linear polarizer to fix the input SOP. The role of the polarization controller is explained in section III. When a pulse propagates down the fiber, it is continuously scattered in all directions via the Rayleigh scattering process [9]. A part of this scattered signal is captured by the fiber and propagates back towards the source. The backscattered signal then passes again through the linear polarizer before reaching the OTDR detector where its power is measured as a function of time. The time scale can be converted into a distance scale so that the POTDR trace provides the backscattered power as a function of the backscattering location as described in more detail in [11] When a plasma current circulates in the vacuum vessel, it generates a magnetic field along the sensing fiber. The component of the magnetic field parallel to the fiber axis generates a circular birefringence via the Faraday effect [7] and induces a SOP rotation. The Faraday-induced birefringence is a nonreciprocal effect so that the direction of the SOP rotation is identical in both directions. In practice, the light SOP varies along an optical fiber even if no magnetic field is applied. A fiber is indeed subject to intrinsic linear birefringence due 


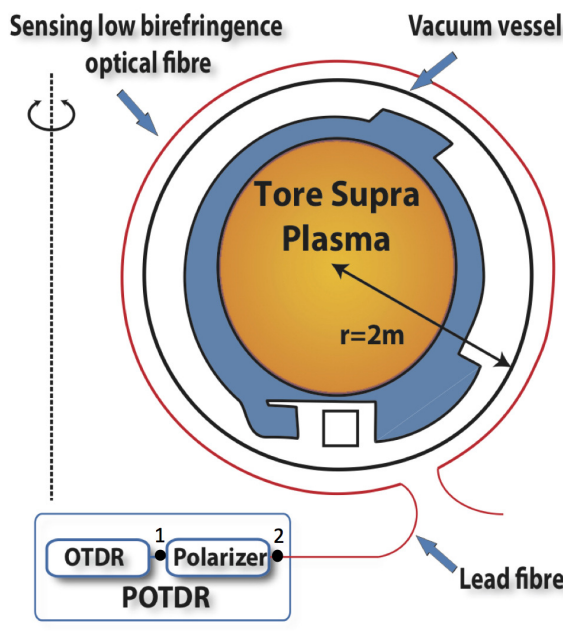

Fig. 1. Section of the vacuum vessel of the Tore Supra tokamak and P-OTDR setup for plasma current monitoring.

to a non perfect circular symmetry of the refractive index distribution in the fiber cross-section. This birefringence adds to the current-induced circular birefringence and therefore constitutes a parasitic effect for current sensing. To minimize the detrimental effect of the intrinsic birefringence, a singlemode low-birefringence fiber (beat length $L_{B}$ of $480 \mathrm{~m}$ ) is used as the sensing fiber in the proposed measurement setup.

The SOP variation along the fiber length is given by $J(z)=\mathbf{M}(z) \mathbf{M}_{\mathbf{L}} J_{\text {in }}$ where $J(z)$ is the normalized Jones vector of the SOP at a distance $z$ starting from the sensing fiber and $J_{i n}$ is the input SOP fixed by the input linear polarizer. $\mathbf{M}_{\mathbf{L}}$ is the Jones matrix of the concatenation of the polarization controller with the lead fiber. Making the hypotheses that the magnetic field is constant (circular vacuum vessel with a central plasma current) and that there are neither twist-induced circular birefringence nor polarization mode coupling (the fiber polarization modes are constant) along the sensing fiber, the backscattered SOP observed at port 2 of the polarizer (see figure 1) after Rayleigh backscattering at a distance $\mathrm{z}$ is given by $J_{B}(z)=\mathbf{M}_{\mathbf{L}}{ }^{T} \mathbf{M}(z) \mathbf{M}(z) \mathbf{M}_{\mathbf{L}} J_{i n}$. As the lead fiber is only characterized by a reciprocal birefringence (no magnetic field), its backward Jones matrix is indeed the transpose of the forward matrix while the hypotheses of no mode coupling and no twist together with the non-reciprocal property of the Faraday effect result to a backward matrix equal to the forward matrix within the sensing fiber as explained in [12]. $\mathbf{M}(z)$ is given by [10]:

$$
\mathbf{M}(z)=\left(\begin{array}{cc}
\alpha+j \beta \cos (2 q) & -\gamma+j \beta \sin (2 q) \\
\gamma+j \beta \sin (2 q) & \alpha-j \beta \cos (2 q)
\end{array}\right)
$$

where

$$
\begin{array}{rlrl}
\alpha=\cos (\Delta z) & \beta & =\frac{\delta}{2} \frac{\sin (\Delta z)}{\Delta} \\
\gamma & =\rho \frac{\sin (\Delta z)}{\Delta} & \Delta & =\sqrt{\rho^{2}+\frac{\delta^{2}}{4}}
\end{array}
$$

$\delta$ is the linear intrinsic birefringence of the fibre given by $\delta=2 \pi / L_{B}$ and $q$ is the angle of its fastest axis. $\rho$ is the circular birefringence induced by the longitudinal magnetic field $B_{l}$ and is given by $\rho=V B_{l}$ [9], yielding:

$$
\rho=\frac{V \mu_{0} I_{P}}{2 \pi r}
$$

where $r$ is the radius of the vacuum vessel circular section and $I_{P}$ the plasma current. $V$ is the Verdet constant equal to $0.54 \mathrm{rad} /(\mathrm{m} . \mathrm{T})$ for standard silica fibers and for a wavelength of $1550 \mathrm{~nm}$ [8]. The backscattered SOP obtained at port 1 of the polarizer is given by $J_{P}(z)=\mathbf{M}_{\mathbf{P}} J_{B}(z)$ where $\mathbf{M}_{\mathbf{P}}$ is the polarizer Jones matrix. Considering the power level backscattered at the polarizer port 2 and the angle of the linear polarizer as the reference power and the reference angle, respectively, yields to a expression of $P_{B}(z)$ (normalized power of the signal measured at the OTDR detector) given by:

$$
P_{B}(z)=r_{1}+r_{2} \cos (4 \Delta z)+r_{3} \sin (4 \Delta z)
$$

where $r_{1}, r_{2}$ and $r_{3}$ are real numbers depending on $\delta, \rho, \mathrm{q}$ and the $\mathbf{M}_{\mathbf{L}}$ matrix coefficients. A more detailed explanation about how equation (5) was obtained can be found in [12].

Based on this theoretical analysis, the proposed plasma current measurement principle consists in four steps: (1) measurement of a POTDR trace, (2) calculation of $P_{B}(z)$ from the POTDR trace by shifting to the linear scale and normalizing by the maximum power, (3) least-squares fitting of $P_{B}(z)$ allowing finding and (4) calculation of $I_{P}$ via equations (3) and (4) considering that $\delta$ is known.

\section{EXPERIMENTAL RESULTS}

The proposed technique was tested on the Tore Supra experimental thermonuclear fusion reactor. As shown in figure 1 , the vacuum vessel is circular with a radius $r=2 \mathrm{~m}$. A low-birefringence fiber was placed around the vacuum vessel. The polarization controller was configured to maximize the backscattered power in the sensing fiber region. The POTDR traces were obtained at $1550 \mathrm{~nm}$ using a pulse duration of 100 ps, a repetition rate of $40 \mathrm{kHz}$ and a peak power of $16.8 \mathrm{~mW}$. A sliding averaging window of $1.89 \mathrm{~m}$ and a time averaging of $10 \mathrm{~s}$ were applied to increase the signal-to-noise ratio of the OTDR traces. After extracting the sensing from the POTDR trace, moving to the linear scale and then, normalizing by the maximum backscatter power, the fitting of the experimental curve by equation (4) is performed to extract $I_{P}$. The value of $\delta$ considered to determine $I_{P}$ is $2 \pi / L_{B}$ where $L_{B}=480 \mathrm{~m}$ (obtained from the low-birefringence fiber datasheet).

The blue trace on figure 2 corresponds to the normalized backscattered power in the sensing fiber distance interval (a more complete trace including the lead fiber can be found in reference [12]) and was obtained for a plasma current set-point of 1.5 MA. One can clearly observe a cosine-like behavior as expected by the theoretical analysis. The green trace corresponds to the fitting by equation (5). The results obtained for various plasma current set-points are shown in Table I. The relative error lies between 1.00 was $13.50 \%$. The worst error was obtained for the smallest $I_{P}$ value for which the ratio between the POTDR signal variation over the measurement distance range and the random noise level is the smallest, leading to a less accurate fitting. 


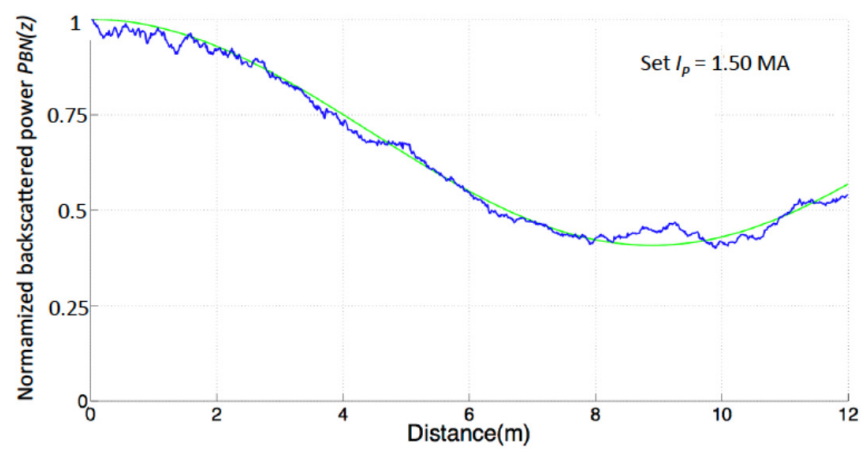

[9] A. J. Rogers, Polarization-optical time domain reflectometry: a technique for the measurement of field distributions, Applied Optics, vol. 20, no. 6, pp. 1060-1074, March 1981.

[10] A. J. Rogers, Y. R. Zhou and V. A. Handerek, Computational Polarization-optical time domain reflectometry for measurement of the spatial distribution of PMD in optical fibres, Proc. Optical Fibre Measurement Conference 1997, pp. 126-129, 1997.

[11] M. Wuilpart, C. Caucheteur et P. Mgret, Measurement of magnetic field using Rayleigh backscattering in optical fibres, Proceed. ANIMMA 2011, 2011.

[12] M. Wuilpart, M. Aerssens, A. Gusarov, Ph. Moreau and P. Mgret, Plasma Current Measurement in Thermonuclear Fusion Reactors Using a Photon-Counting POTDR, Photonics Technology Letters, vol. 29, no. 6 , pp. 547-550, March 2017

Fig. 2. Normalized POTDR trace in the sensing region.

\begin{tabular}{|c|c|c|}
\hline Set $I_{P}(\mathrm{MA})$ & Measured $I_{P}(\mathrm{MA})$ & Relative error(\%) \\
\hline 1.5 & 1.475 & 1.67 \\
\hline 1.3 & 1.357 & 4.38 \\
\hline 1.2 & 1.248 & 4.00 \\
\hline 1.0 & 0.999 & 1.00 \\
\hline 0.8 & 0.791 & 1.13 \\
\hline 0.7 & 0.693 & 1.00 \\
\hline 0.6 & 0.519 & 13.50 \\
\hline \multicolumn{3}{|c|}{ TABLE I }
\end{tabular}

EXPERIMENTAL RESULTS

\section{CONCLUSION}

We presented a fiber-optic current sensor for the measurement of plasma current in thermonuclear fusion reactors. The sensor includes a low-birefringence fiber placed around a the vacuum vessel. The interrogator consists of a POTDR unit. The plasma current can be calculated after fitting the experimental trace and removing the contribution of the intrinsic linear birefringence. The sensor has been experimentally validated on the Tore Supra fusion reactor for a plasma current range going from 0.6 MA to 1.5 MA.

\section{REFERENCES}

[1] D. van Houtte, G. Martin, A. Bécoulet, J. Bucalossi, G. Giruzzi, G.T. Hoang, Th. Loarer, B. Saoutic and the Tore Supra Team, Recent fully non-inductive operation results in tore supra with $6 \mathrm{~min}, 1 \mathrm{gj}$ plasma discharges, Nuclear Fusion, vol. 44, no. 5, L11, April 2004.

[2] Chen Qing, Li Hong-bin, Huang Ben-xiong and Dou Qiao-qi., Rogowski sensor for plasma current, Sensors Journal, IEEE, vol. 9, no. 3, pp. 293296, March 2009.

[3] R. Van Nieuwenhove and L. Vermeeren, Study of the radiation induced electromotive force effect on mineral insulated cables for magnetic diagnostics in ITER, Fusion Engineering and Design, 22nd Symposium on Fusion Technology, vol. 66-68, pp. 821-825, September 2003.

[4] Ph. Moreau, B. Brichard, A. Fil, Ph. Malard, P. Pastor, A. Le-Luyer, F. Samaille and V. Massaut, Test of fiber optic based current sensors on the tore supra tokamak, Fusion Engineering and Design, vol. 86, no. 6-8, pp. 1222-1226, October 2011.

[5] L. Palmieri, D. Sarchi and A. Galtarossa, Distributed measurement of high electric current by means of polarimetric optical fiber sensor, Optics Express, vol. 23, no. 9, pp. 11073-11079, April 2015.

[6] M. Aerssens, F. Descamps, A. Gusarov, P. Mégret, P. Moreau and M. Wuilpart, Influence of the optical fiber type on the performances of fiber-optics current sensor dedicated to plasma current measurement in ITER, Applied Optics, vol. 54, no. 19, pp. 5983-5991, July 2015.

[7] T. Yoshino, Theory for the Faraday effect in optical fiber, J. Opt. Soc. Am. B, vol. 22, no. 9, pp. 1856-1860, September 2005

[8] M. V. Andres, J. L. Cruz and M. A. Hernandez, Faraday effect in standard optical fibers: dispersion of the effective verdet constant, Applied Optics, vol. 35, no. 6, pp. 922-927, February 1996. 